\title{
The Drosophila Brahma complex is an essential coactivator for the trithorax group protein Zeste
}

\author{
Arnoud J. Kal, ${ }^{1}$ Tokameh Mahmoudi, ${ }^{1}$ Naomi B. Zak, ${ }^{2}$ and C. Peter Verrijzer ${ }^{1,3}$ \\ ${ }^{1}$ Department of Molecular and Cell Biology, MGC, Centre for Biomedical Genetics, Leiden University Medical Centre, 2300 \\ RA Leiden, The Netherlands and Imperial Cancer Research Fund, London WC2A 3PX, UK; ${ }^{2}$ Experimental Medicine \\ and Cancer Research, Hebrew University, Hadassah Medical School, Jerusalem 91120, Israel
}

The trithorax group (trxG) of activators and Polycomb group (PcG) of repressors are believed to control the expression of several key developmental regulators by changing the structure of chromatin. Here, we have sought to dissect the requirements for transcriptional activation by the Drosophila trxG protein Zeste, a DNA-binding activator of homeotic genes. Reconstituted transcription reactions established that the Brahma (BRM) chromatin-remodeling complex is essential for Zeste-directed activation on nucleosomal templates. Because it is not required for Zeste to bind to chromatin, the BRM complex appears to act after promoter binding by the activator. Purification of the Drosophila BRM complex revealed a number of novel subunits. We found that Zeste tethers the BRM complex via direct binding to specific subunits, including trxG proteins Moira (MOR) and OSA. The leucine zipper of Zeste mediates binding to MOR. Interestingly, although the Imitation Switch (ISWI) remodelers are potent nucleosome spacing factors, they are dispensable for transcriptional activation by Zeste. Thus, there is a distinction between general chromatin restructuring and transcriptional coactivation by remodelers. These results establish that different chromatin remodeling factors display distinct functional properties and provide novel insights into the mechanism of their targeting.

[Key Words: Brahma; Zeste; chromatin; transcription; Trithorax group; SWI/SNF]

Received November 15, 1999; revised version accepted March 17, 2000.

The establishment of the basic body plan of higher eukaryotes is dependent on the spatially restricted expression patterns of homeotic genes (McGinnis and Krumlauf 1992). In the Drosophila embryo the boundaries of expression of the homeotic genes are established by transiently expressed regulators and maintained by the trithorax group (trxG) and Polycomb group (PcG) proteins (Kennison 1995; Paro 1995; Kingston et al. 1996; Pirrotta 1998; Lyko and Paro 1999|. The PcG proteins are required to preserve the transcriptionally silenced state, whereas the trxG proteins are needed to perpetuate the transcriptionally active state. The function of these factors is not limited to homeotic gene regulation; instead, they are involved in the control of diverse developmental processes (Phillips and Shearn 1990; Pelegri and Lehmann 1994; Breen et al. 1995; Brizuela and Kennison 1997; Elfring et al. 1998; Jacobs et al. 1999; Vazquez et al. 1999). The molecular mechanisms by which trxG and PcG proteins act remain largely unclear. However, several observations suggest that they change the structure of chromatin, establishing a configuration that is either permissive or nonpermissive for transcription (Kingston

${ }^{3}$ Corresponding author.

E-MAIL verrijzer@lumc.nl; FAX (31) 71-527-6284. et al. 1996; Pirrotta 1998; Lyko and Paro 1999; Shao et al. 1999).

The packaging of DNA into chromatin generates a barrier to processes that require access to the DNA such as transcription (Armstrong and Emerson 1998; Kadonaga 1998; Workman and Kingston 1998; Kingston and Narlikar 1999; Kornberg and Lorch 1999). One of the main strategies by which cells alleviate chromatin-mediated repression is through the action of ATP-dependent chromatin remodeling complexes (Tsukiyama and $\mathrm{Wu} 1997$; Varga-Weisz and Becker 1998; Workman and Kingston 1998; Kingston and Narlikar 1999; Travers 1999; Wade and Wolffe 1999). The founding member of this evolutionarily highly conserved family of factors is the yeast multisubunit SWI/SNF complex. Its catalytic subunit is the Swi2p/Snf2p DNA-stimulated ATPase, which, like several other SWI/SNF subunits, was identified by genetic screens for regulators of transcription (Winston and Carlson 1992; Tamkun 1995).

The Drosophila homolog of the yeast SWI2/SNF2 gene, brahma (brm), was isolated as a dominant suppressor of $P c$ mutations and therefore belongs to the trxG (Kennison and Tamkun 1988; Tamkun et al. 1992). BRM is part of a large multiprotein complex containing several other proteins with similarity to subunits of yeast 
SWI/SNF or the related RSC (remodels the structure of chromatin) complex (Papoulas et al. 1998; Kingston and Narlikar 1999). Mammalian SWI/SNF or Brahma-associated factors (BAF) complexes contain both conserved and unique subunits, suggestive of functional differentiation (Wang et al. 1996; Armstrong and Emerson 1998; Workman and Kingston 1998). One of the human BAF complexes, E-RC1, is required for transcriptional activation by erythroid kruppel-like factor (EKLF), a tissue-specific regulator of the $\beta$-globin gene (Armstrong et al. 1998).

Another class of multisubunit remodeling factors is characterized by the presence of the ISWI ATPase as a catalytic core (Cairns 1998; Kadonaga 1998; Varga-Weisz and Becker 1998). A number of distinct ISWI-containing remodelers, comprising NURF (nucleosome-remodeling factor; Tsukiyama and Wu 1995), chromatin-accessibility complex (CHRAC; Varga-Weisz et al. 1997), and ACF (ATP-utilizing chromatin assembly and remodeling factor; Ito et al. 1997), have been isolated from Drosophila embryos using different functional assays. Reconstitution experiments revealed that in both the SWI/SNF and ISWI remodeling factors, the engine of the complex is formed by the ATPase. However, association with other subunits modulates the functionality of the complexes and can greatly enhance their activity /Corona et al. 1999; Hamiche et al. 1999; Ito et al. 1999; Längst et al. 1999; Phelan et al. 1999; Travers 1999).

Although the in vivo role of trxG proteins in the control of gene expression is well established, our understanding of the mechanisms of action of trxG proteins has been hampered by a lack of biochemical information. The extensive homology of the BRM complex to SWI/ SNF strongly suggests that it is also a chromatin remodeling factor. The majority of trxG proteins, however, are not associated with BRM and appear to function in a distinct manner (Papoulas et al. 1998). An exception is MOR (Kennison and Tamkun 1988; Brizuela and Kennison 1997), which is homologous to yeast Swi3p and was recently identified as a BRM associated protein (BAP; Crosby et al. 1999). Interestingly, osa/eyelid (osa) shows a strong genetic interaction with brm, suggesting that its gene product may closely cooperate with the BRM complex (Treisman et al. 1997; Vazquez et al. 1999). Finally, Zeste and GAGA are sequence-specific transcription regulators, which form large homo-oligomers that bind cooperatively to the multiple sites present in their natural response elements (Chen and Pirrotta 1993; Katsani et al. 1999).

Zeste is an intriguing protein that controls gene expression in surprisingly different ways (Rosen et al. 1998). Firstly, it is a DNA-binding activator of homeotic and other genes (Biggin et al. 1988; Laney and Biggin 1992). On polytene chromosomes of the larval salivary gland, Zeste is found associated with over 60 different sites, which is consistent with a role in the regulation of many genes (Pirrotta et al. 1988; Rastelli et al. 1993). Secondly, Zeste loss-of-function mutations are enhancers of position-effect variegation suggesting that Zeste counteracts heterochromatin-induced silencing (Judd 1995). Thirdly, Zeste mediates transvection, and particu- lar gain-of-function mutations turn Zeste into a pairingdependent repressor of gene expression (Rosen et al. 1998). Finally, Zeste shows positive as well as negative allele-specific genetic interactions with several PcG genes (Phillips and Shearn 1990; Pelegri and Lehmann 1994). Thus, Zeste performs a variety of distinct functions during chromatin-directed gene regulation.

An interesting question is how trxG proteins and chromatin remodeling factors act in a gene-specific manner. One possibility is that they are recruited to transcription control regions by sequence-specific activators. Here, we address this issue using a natural regulator of transcription, the trxG protein Zeste. Biochemical complementation experiments revealed that the BRM complex is an essential chromatin-specific coactivator for Zeste. Moreover, purification of the BRM complex provided evidence for the existence of novel BAPs, including another trxG protein. We compared the functional properties of the BRM complex with those of ISWI remodelers using in vitro transcription and nucleosome spacing assays. Results from these experiments revealed a functional specialization among distinct chromatin remodelers. Finally, protein-protein interaction assays were used to identify targets for Zeste within the BRM complex. Our findings suggest that the BRM complex is a transcriptional coactivator that can be recruited to specific genes by Zeste.

\section{Results}

Zeste directs remodeling and transcription on chromatin templates

To examine the role of chromatin structure in transcriptional activation by Zeste, we set out to reconstitute Zeste-directed transcription on nucleosomal templates. FLAG epitope-tagged Zeste was expressed in Sf9 cells infected with recombinant baculoviruses and purified to near homogeneity by immunoaffinity chromatography (Fig. 1A). To test the ability of Zeste to activate transcription on chromatin, we assembled a plasmid containing five Zeste-binding sites next to a core promoter into a nucleosomal template. The number of binding sites chosen was based upon previous results showing that Zeste requires at least four binding sites for efficient DNA binding. Indeed, natural Zeste response elements typically contain five or more Zeste sites (Biggin et al. 1988; Chen and Pirrotta 1993).

Chromatin templates were generated by two different methods: either by using the Drosophila embryo-derived S-190 assembly system (Kamakaka et al. 1993) or by a salt dialysis protocol. Incubation of the plasmid with purified Drosophila core histones and the S-190 extract resulted in the assembly of long arrays of physiologically spaced nucleosomes (see Fig. 1D for an example). After assembly, these templates were treated with sarcosyl and purified over a sucrose gradient to remove proteins not associated with chromatin. Alternatively, chromatin was assembled by salt gradient dialysis followed by purification over a sucrose gradient. The salt dialysis 
Kal et al.

Figure 1. Zeste directs remodeling and transcription on nucleosomal arrays. (A) Recombinant FLAG-tagged Zeste was expressed in baculovirus infected Sf9 cells and, after extract preparation, immunopurified on an anti-FLAG column. The Zeste protein fractions and the purified Drosophila core histones were analyzed by SDS-PAGE followed by Coomassie blue staining. The positions of Zeste, core histones, and the molecular masses $(\mathrm{kD})$ of protein standards are indicated. $(B)$ Outline of the experimental design of the transcription experiments described in this article. Nucleosomal templates were generated using either the Drosophila embryo assembly system or by salt gradient dialysis. After sarcosyl treatment, in the case of S-190 assembly, and purification by sucrose gradient sedimentation, the templates were used in reconstituted transcription reactions. The transcription machinery and chromatin remodeling factors were provided by a partially purified nuclear extract (H0.4). Following a preincubation of $20 \mathrm{~min}$ in the presence (or absence, see Fig. 1F) of ATP and either no activator or purified Zeste, transcription was started by the addition of the remaining NTPs and allowed to proceed for $30 \mathrm{~min}$. Transcription products were visualized by primer extension. (C) Transcriptional activation by Zeste. Transcription on chromatin templates (plasmid pAK156) generated using either the S-190 assembly system (lanes 1,2) or by salt gradient dialysis (lanes 3,4) was tested either in the absence (lanes 1,3 ) or presence of Zeste (lanes 2,4). (D) Zeste directs localized chromatin remodeling. S-190 assembled chromatin templates lacking (lanes 1,3) or bound by Zeste (lanes 2,4) were digested with MNase and analyzed by Southern blot hybridization using oligonucleotides corresponding to either promoter sequences (lanes 3,4) or distal plasmid sequences (the Amp gene; lanes 1,2).
A

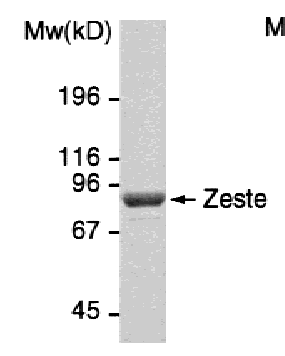

$\mathrm{Mw}(\mathrm{kD})$

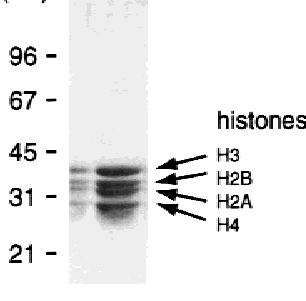

B

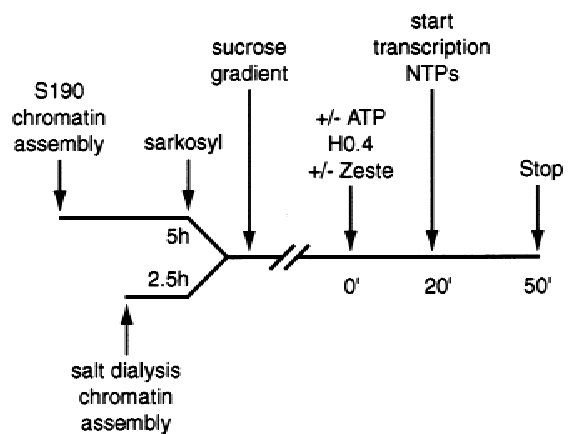

C

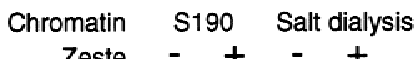

Zeste

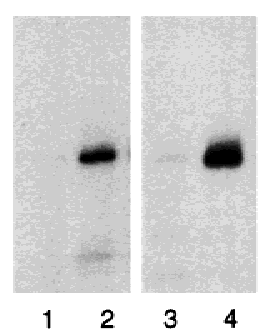

D

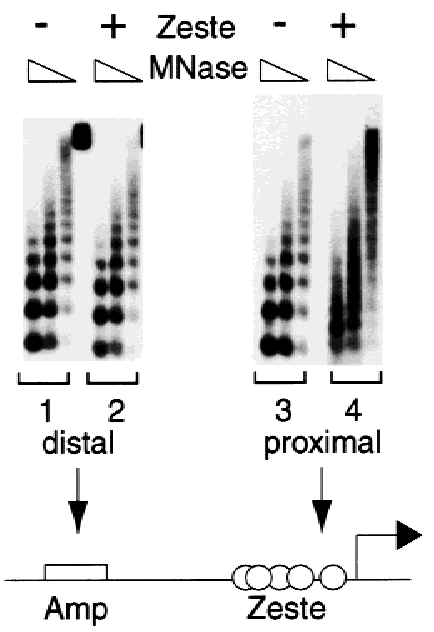

E

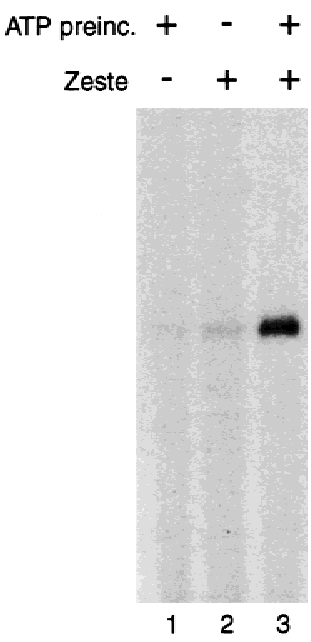

(E) ATP-dependent remodeling is a prerequisite for Zeste activation. A nuclear extract (H0.4) with either no activator (lane 1) or Zeste (lanes 2,3) was added to chromatin templates assembled by salt dialysis and purified over a sucrose gradient. Following a 20 min incubation in the presence (lanes 1,3) or absence of ATP (lane 2), transcription was initiated by the addition of NTPs. The analysis of transcription was performed as described in Fig. 1B.

method does not yield the long periodic arrays achieved with the S-190 system (see Fig 4B for an example). However, because the input comprises only the DNA template and highly purified histones (Fig. 1A), the resulting chromatin is completely defined. Thus, each method of assembly has some advantages that complement the other.

The two types of nucleosomal templates were used in reconstituted transcription experiments as outlined in Figure 1B. The general transcription machinery and chromatin-remodeling factors were provided by a partially purified Drosophila embryo nuclear extract (H0.4). The nucleosomal templates assembled with the S-190 system or by salt dialysis were transcriptionally inert in the absence of Zeste (Fig. 1C, lanes 1,3). Addition of Zeste resulted in a strong activation of transcription on both chromatinized templates (Fig. 1C, lanes 2,4). As ex- pected, activation by Zeste was critically dependent on the presence of Zeste-binding elements (data not shown).

We next asked whether activation of transcription by Zeste would involve the generation of an open chromatin structure. To investigate this possibility, we digested the nucleosomal templates with MNase followed by gel electrophoresis and transfer to a nitrocellulose membrane. Indeed, hybridization of the membrane with a probe for promoter sequences revealed a dramatic Zestedependent disruption of the chromatin structure (Fig. 1D, lanes 3,4$)$. The remodeling is strictly localized, because reprobing of the blot with a probe corresponding to a distal region showed no disruption of the nucleosome array in the presence of Zeste (Fig. 1D, lanes 1,2). To establish how far the chromatin disruption spreads, we repeated this experiment using oligonucleotides corresponding to distinct promoter areas. These experiments 
indicated that the chromatin remodeling spreads $\sim 100 \mathrm{bp}$ beyond the Zeste-binding sites and include the core promoter area (data not shown).

It has been well established that localized chromatin disruption by sequence-specific DNA-binding proteins involves the action of ATP-dependent remodeling factors. Indeed, we observed that Zeste-directed disruption of the nucleosomal array required the presence of ATP (data not shown). We next asked whether ATP-dependent remodeling was a prerequisite for transcriptional activation on chromatin by Zeste (Fig. 1E). To test this hypothesis, Zeste and a nuclear extract (H0.4) were added to a chromatin template, and after an incubation of $20 \mathrm{~min}$, either in the absence (Fig. 1E, lane 2) or presence (Fig. 1E, lanes 1,3) of ATP, transcription was assayed. High levels of transcription required a preincubation in the presence of both ATP and Zeste. We conclude that Zeste is a potent activator of transcription on chromatin and may require the action of an energy consuming remodeling factor.

\section{The BRM complex, but not the ISWI remodelers, is} required for activation by Zeste

We next searched for such a possible ATP-dependent cofactor for Zeste activation. In parallel to a biochemical fractionation approach, we considered the known Drosophila chromatin remodelers. Three distinct remodeling factors have been isolated from fly embryo extracts: NURF, ACF, and CHRAC (Tsukiyama and Wu 1995; Ito et al. 1997; Varga-Weisz et al. 1997). Interestingly, all three complexes contain the ISWI protein as a common subunit (Cairns 1998; Kingston and Narlikar 1999). In addition, although no biochemical activities have been described for the BRM complex, its high similarity to yeast and human SWI/SNF stongly suggest that it is also a remodeling factor (Tamkun et al. 1992; Papoulas et al. 1998). To determine whether any of these Drosophila remodeling factors are required for Zeste function, we took an immunodepletion approach utilizing affinity-purified antibodies against either BRM or ISWI. The nuclear extracts used in these experiments were generated by a procedure similar to that used to prepare extracts containing ACF, NURF, or CHRAC (see Materials and Methods). Importantly, essentially all the ISWI and BRM present in the nuclear extract is retained in the H0.4 fraction as judged by Western blotting analysis with antibodies directed against either ISWI or BRM (data not shown).

We next tested whether depletion of either the BRM complex or the ISWI-remodelers would affect the abillity of Zeste to bind to S-190 assembled chromatin templates (Fig. 2A). DNase I footprinting analysis revealed efficient binding of Zeste to its binding sites in the transcription template, either present as naked DNA (Fig. 2A, lanes $1,2)$ or assembled into chromatin (Fig. 2A, lanes 3-8). Interestingly, neither the depletion of the BRM complex (Fig. 2A, lanes 5,6) nor depletion of the ISWI complexes (Fig. 2A, lanes 7,8) affected Zeste binding. Moreover, at
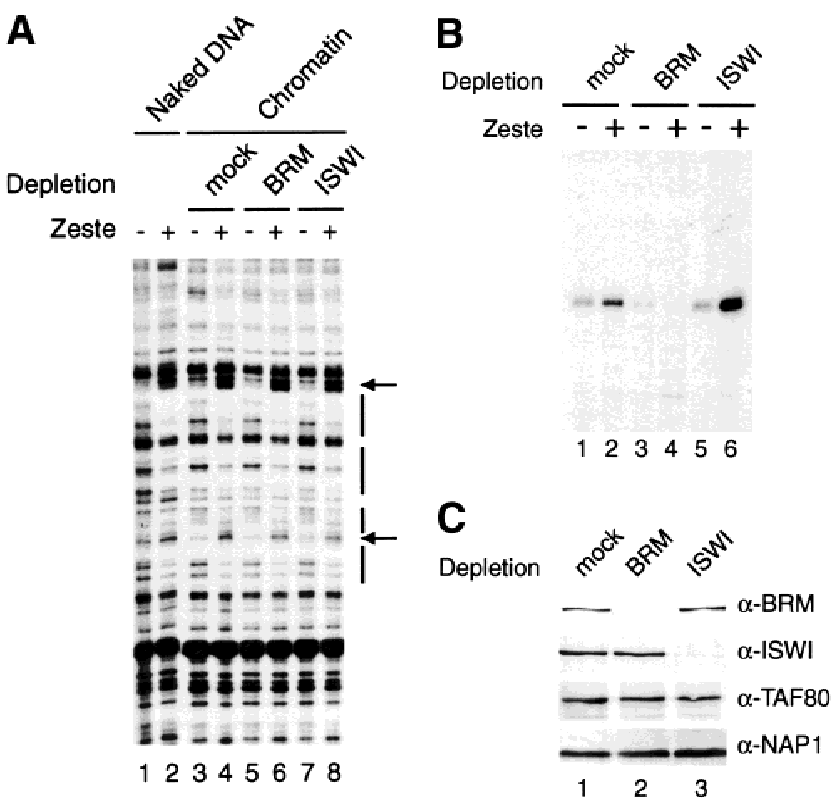

Figure 2. Activation by Zeste requires the BRM complex, but not the ISWI factors. (A) DNaseI footprinting analysis of Zeste on either naked DNA (lanes 1,2) or on S-190 assembled chromatin (plasmid pAK156) using extracts that were either mockdepleted (lanes 3,4) or depletion with affinity purified anti-BRM antibodies (lanes 5,6) or anti-ISWI antibodies (lanes 7,8). After completion of assembly, chromatin was incubated for $1 \mathrm{hr}$ either in the absence (odd numbered lanes) or presence of Zeste (even numbered lanes) followed by digestion with DNaseI. For digestion of the chromatin template, an 150-fold higher amount of DNaseI was used than for naked DNA. The DNaseI digestion pattern was visualized by primer extension. The Zeste footprints and DNaseI hypersensitive sites are indicated with bars and arrows, respectively. $(B)$ The ability of H0.4 partially purified nuclear extract to support Zeste activation was tested after either mock depletion (lanes 1,2), depletion with affinity purified anti-BRM antibodies (lanes 3,4) or anti-ISWI antibodies (lanes 4,6$)$. The chromatin templates were assembled using the S-190 system, sarcosyl treated and purified over a sucrose gradient prior to use in transcription reactions either in the absence (odd numbered lanes) or presence of Zeste (even numbered lanes). The experimental design was as described in Fig. 1B. (C) The efficiency and specificity of the immunodepletion of the H0.4 fraction with either mock (lane 1), anti-BRM (lane 2), or anti-ISWI antibodies (lane 3) was verified by Western blot analysis using antibodies directed against BRM, ISWI, $\operatorname{dTAF}_{\text {II }} 80$, or NAP-1.

least in these extracts, the binding affinity of Zeste for chromatin templates was comparable to that for naked DNA. We then asked whether the immunodepleted transcription extracts were able to support activation by Zeste on chromatin (Fig. 2B). Strikingly, depletion of the BRM complex abolished activation of transcription by Zeste (Fig. 2B, lanes 3,4), whereas depletion of the ISWIcontaining complexes had no effect (Fig. 2B, lanes 5,6). The depletion of Brahma or ISWI was efficient and specific and did not affect other factors involved in transcription and chromatin dynamics such as TAF80 or NAP1 (Fig. 2C). 
Kal et al.

These results indicate that the BRM complex, but none of the ISWI remodelers, is essential for activation by Zeste. Thus, different remodeling complexes display distinct regulatory activities. Moreover, the observation that the BRM complex is not required for Zeste binding to the chromatin template, suggests that it functions subsequent to promoter binding by the activator. Because of its apparent importance for Zeste function, we set out to purify the Drosophila BRM complex.

\section{Purification and characterization of the BRM complex}

The BRM complex was purified from fly embryo nuclear extracts by conventional column chromatography, guided by Western blot analysis of fractions with antibodies against BRM. The steps in the purification of the endogenous BRM complex are outlined in Figure 3A. The BRM-containing fractions from the final monoS column were analyzed by SDS-PAGE and visualized by silver staining (Fig. 3B). The identity of BRM and MOR was confirmed by Western blot analysis (Fig. 3B,C). We observed a number of major bands that copurify with BRM. Several, but not all, of these polypeptides corresponded to the pattern described for the BRM complex by
Tamkun and coworkers (Papoulas et al. 1998). For example, we noted a doublet with a molecular weight of approximately $300 \mathrm{kD}$ (p300), a diffuse band estimated at $400 \mathrm{kD}$ as well as as several other polypeptides. Because we purified the BRM complex from embryo extracts up to $12 \mathrm{hr}$, it is possible that substoichiometric tissue-specific BRM complexes are present in these fractions. Such a heterogeneity has previously been reported for human BAF complexes (Wang et al. 1996).

Recently, a strong genetic interaction between brm and another trxG gene, osa, has been described (Vazquez et al. 1999). Because the OSA protein has a predicted molecular weight of $300 \mathrm{kD}$, we reasoned that it would be an attractive candidate for the p300 BAP. To test this idea, we used a monoclonal antibody directed against OSA (Treisman et al. 1997) for Western blot analysis of the purified BRM complex (Fig. 3C). We compared a crude nuclear fraction (H0.4) with a peak fraction from the final column in our BRM complex purification scheme. The anti-OSA antibody efficiently recognized p300 as well as a number of bands migrating between 200 and $300 \mathrm{kD}$, which might be alternatively processed forms (Fig. 3C, lanes 1,2). The BRM and MOR subunits were readily detected by Western blot analysis (Fig. 3C, lanes 3-6), whereas there was no detectable ISWI protein
Figure 3. Purification and characterization of the BRM complex. (A) Outline of the chromatographic scheme used to purify the Drosophila BRM complex. (B) Polypeptide composition of BRM-containing fractions from the final monoS column. Proteins were resolved by SDS-PAGE and visualized by silver staining (top) or Western blotting with an anti-BRM antibody (bottom). (C) Western blot analysis of the purified BRM complex. Nuclear extract (H0.4, odd numbered lanes) and a peak fraction from the monos column (43, even numbered lanes) were analyzed by SDS-PAGE followed by Western blotting using antibodies directed against OSA (lanes 1,2), BRM (lanes 3,4), MOR (lanes 5,6), and ISWI (lanes 7,8). (D) Identification of the core BRM complex. A peak fraction from the monoS column (\#42, lane 1) was incubated with beads coated with affinity purified anti-BRM antibodies. Proteins retained on the beads after extensive washes with a buffer containing $800 \mathrm{~mm} \mathrm{KCl}$ and $0.01 \%$ NP-40, were resolved by SDS-PAGE on either an $8 \%$ polyacrylamide gel (lane 2 ) or a $12 \%$ polyacrylamide gel (lane 3 ) and stained with silver. (E) OSA is part of the core BRM complex. The input fraction (monoS \#42, lane 1), bound material after the $800 \mathrm{~mm}$ $\mathrm{KCl}$ washes of the immunoprecipitation with antiBRM beads (lane 2) and unbound material (lane 3), were analyzed by SDS-PAGE on a $6 \%$ polyacrylamide gel followed by Western blotting using a monoclonal antibody directed against OSA.
A

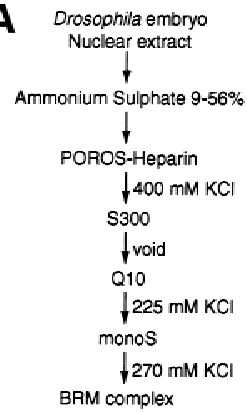

B monoS chromatography
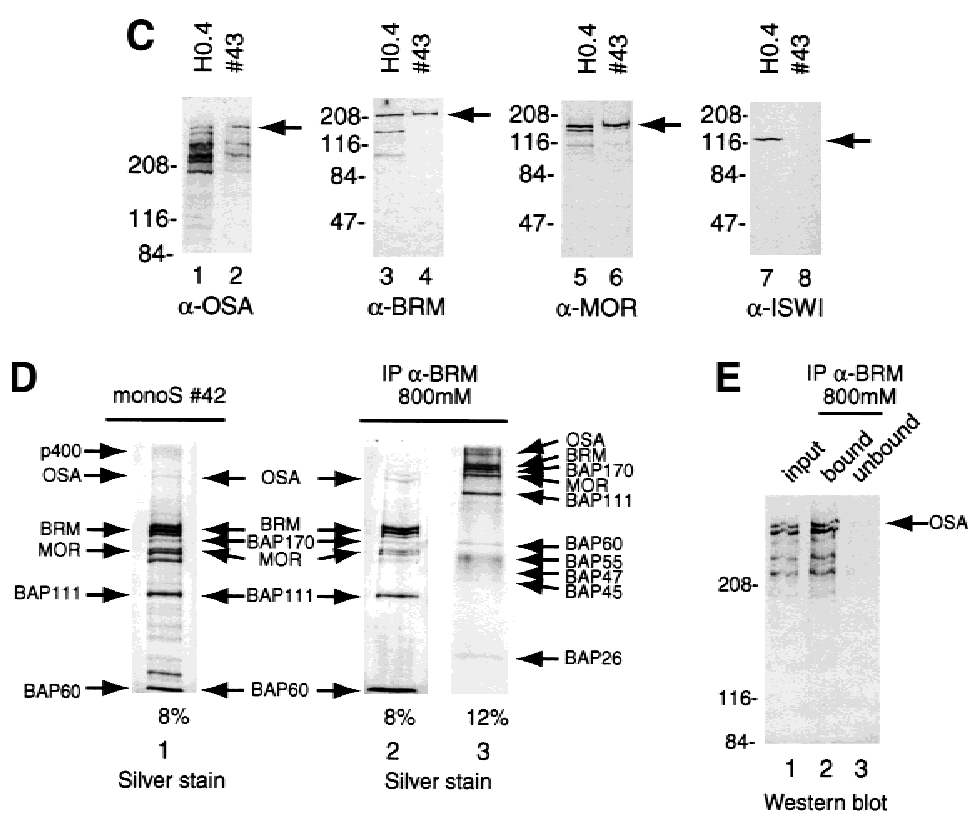
present in the purified BRM complex (Fig. 3C, cf. lane 7 with lane 8). Furthermore, Zeste and components of the general transcription machinery such as RNA polymerase II, TFIID, or NAP1 were also absent (data not shown).

\section{TrxG protein OSA is an integral part of the BRM complex}

Having identified p300 as OSA, we next wished to establish how tightly this protein and the other potential BAPs were associated with the BRM complex. Therefore, we repeated the immunoprecipitation on the $270 \mathrm{~mm}$ $\mathrm{KCl}$ monoS fraction. The bound complex was then extensively washed with a buffer containing $800 \mathrm{~mm} \mathrm{KCl}$ and NP40. Proteins that precipitated with the anti-BRM beads were analyzed by SDS-PAGE followed by silver staining (Fig. 3D). Several polypeptides dissociated under these stringent conditions. However, it should be noted that most of these proteins, including p400, remain associated with the BRM complex when less stringent conditions were used ( $250 \mathrm{~mm} \mathrm{KCl}$; data not shown). Thus, there may be several factors that associate more loosely with the core BRM complex. The BRM core complex appears to comprise 10 polypeptides including BRM, MOR, BAP111, BAP60, BAP 55, BAP 47, and BAP45. We did not observe a protein corresponding to BAP74 (Papoulas et al. 1998). Interestingly, we detected three novel BAPs comprising OSA, a $170-\mathrm{kD}$ and a $26-\mathrm{kD}$ protein. The identity of the OSA protein in the core BRM complex was confirmed by Western blot analysis (Fig. 3E). It should be noted that the light staining MOR band (Fig. $3 \mathrm{E}$, lane 1) runs very close to a polypeptide that is not stably associated with the BRM complex (Fig. 3E, lane 2). Western blot analysis with distinct antibodies was used to identify BRM and MOR (data not shown). Taken together, these data show that the BRM complex consists of a core of 10 tightly associated subunits, including the trxG protein OSA, and several more loosely associated factors.

The BRM complex is a chromatin-specific coactivator of Zeste

Our depletion studies suggested that the BRM complex is required for transcriptional activation by Zeste on chromatin. We next wanted to test whether activation could be restored by the addition of the highly purified BRM complex (Fig. 4A). In these experiments, we used three different templates: (1) chromatin assembled with the fly embryo S-190 assembly system, treated with sarcosyl, and purified over a sucrose gradient; (2) chromatin assembled with pure histones by salt dialysis and purified by sucrose gradient sedimentation; and (3) naked DNA. The transcription system was either mock depleted (Fig. 4A, lanes 1,2) or depleted for the BRM complex (Fig. 4A, lanes 3-6). Similar to our previous experiments, depletion of the BRM complex abolished activation by Zeste on chromatin templates. Zeste-directed transcription was completely restored by the addition of

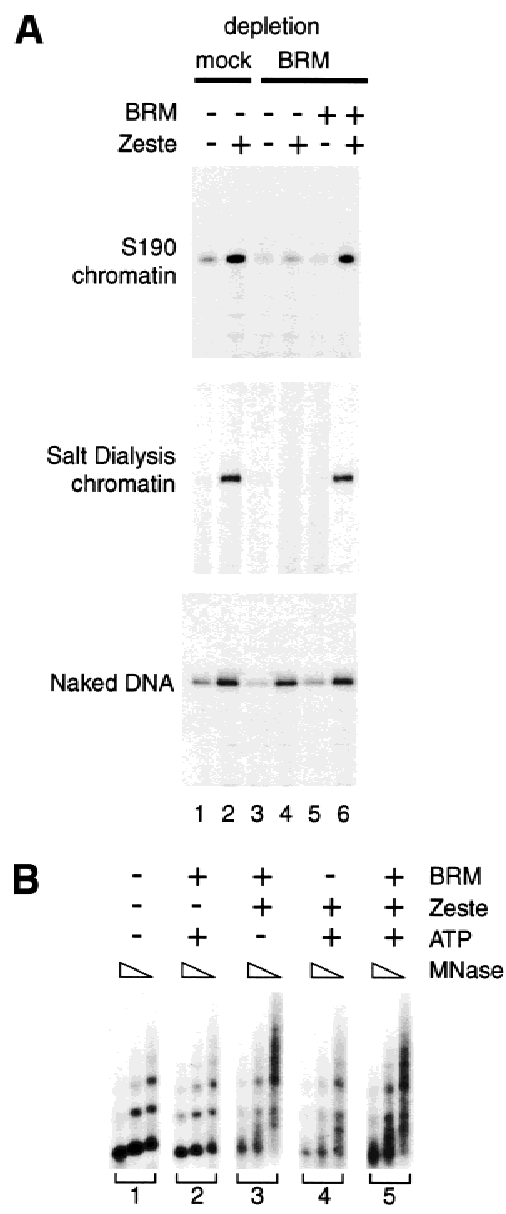

Figure 4. BRM complex mediates chromatin-specific transcriptional activation by Zeste. $(A)$ The ability of the purified BRM complex (monoS \#42) to restore Zeste-directed transcription in a BRM-depleted transcription system (lanes 3-6) was tested using either S-190 assembled chromatin (top), salt dialysis assembled chromatin (middle), or naked DNA (bottom) as a template. Mock-depleted H0.4 extract was used as a positive control (lanes 1,2). Transcription reactions were either in the absence (odd numbered lanes) or presence of Zeste (even numbered lanes). Approximately 20 fmoles of BRM complex was added to reactions 5 and 6 in which the nucleosome to BRM ratio was $\sim 50: 1$. The experimental design was essentially as described in Fig. 1. (B) Zeste-directed remodeling in a defined system. The Zeste-responsive template was assembled into chromatin by salt dialysis and incubated in the presence of varying combinations of BRM complex (about 20 femtomoles), Zeste, and ATP. After $30 \mathrm{~min}$, the templates were digested with MNase and analyzed by Southern blot hybridization using an oligonucleotide corresponding to part of the promoter sequence.

the purified BRM complex. On the S-190 chromatin, addback of the BRM complex resulted in an 20 -fold stimulation of Zeste activation, whereas on the salt-dialysis chromatin the stimulation was about 40 -fold. We estimated that in these transcription reactions the BRM complex is present at a BRM/nucleosome molar ratio of at most 1 to 50, as judged by comparing these factors with proteins of known concentration on silver and Coo- 
massie-stained gels. Importantly, the coactivator function of the BRM complex is strictly chromatin-specific since activation by Zeste on naked DNA was independent of the BRM complex. We also tested the ability of Zeste to induce chromatin remodeling in a minimally defined system. Chromatin was assembled by salt dialysis using pure histones (Fig. 4B). Next, highly purified Zeste, BRM complex, and ATP were added in various combinations. After $30 \mathrm{~min}$, the structure of the nucleosomal array was probed by MNase-digestion followed by Southern blot analysis using an oligonucleotide complementary to DNA close to the Zeste binding sites. Surprisingly, Zeste by itself already induced changes in the MNase ladder consistent with the notion that it can interact with chromatin in the absence of the BRM complex. Moreover, addition of BRM complex and ATP, did not induce a dramatically stronger reconfiguration of the chromatin structure. As expected, there was no disruption of the chromatin structure at a distal location (data not shown). We conclude that the BRM complex is an essential chromatin-specific coactivator of Zeste.

\section{Role of BRM and ISWI remodelers in nucleosomal spacing}

In addition to their involvement in transcription regulation, remodeling factors participate in the formation of periodic nucleosome arrays. In particular, the ability of several ISWI-containing complexes to mediate nucleosome spacing has been well established (Ito et al. 1997; Varga-Weisz et al. 1997; LeRoy et al. 1998; Kingston and Narlikar 1999; Tsukiyama et al. 1999). To ensure that the failure of the ISWI factors to support Zeste-directed transcription was not due to their inactivation, we tested our ISWI fraction in a nucleosome spacing assay. Poorly spaced nucleosomal arrays were assembled using a modified salt dialysis protocol. As expected, a partially purified ISWI fraction (monoS column, see Materials and Methods), containing the majority of the ISWI present in the nuclear extract, efficiently converted irregular chromatin into a nucleosomal array with periodic spacing in an ATP-dependent manner (Fig. 5, lanes 1-3). Importantly, the amount of ISWI that could efficiently order bulk chromatin, failed to support Zeste-dependent transcription in BRM-depleted transcription reactions (Fig. 4; data not shown). Thus, these experiments discriminate between general chromatin modulation and transcriptional coactivation. Moreover, the ability of the BRM complex, but not the ISWI factors, to support activation by Zeste establishes a functional differentiation between distinct remodeling factors. In contrast to the fraction containing approximately equal molar amounts of ISWI, the BRM complex displays only weak nucleosome spacing activity (Fig. 5, lanes 4-6). Moreover, when the relative protein concentrations of the remodeling factors were varied, we consistently observed that the ISWI fraction is a more potent spacing factor than the BRM complex. Although, in these experiments we do not discriminate between distinct ISWI-containing complexes, these

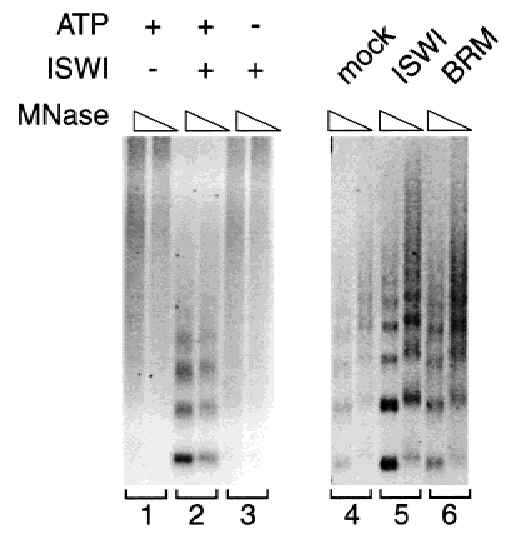

Figure 5. Comparison of the nucleosome spacing activity of the ISWI factors and the BRM complex. Suboptimally spaced nucleosomal DNA was prepared by a stepwise salt dilution and dialysis protocol using pure histones. To test for ATP-dependent nucleosome spacing activity, these templates were incubated for $1 \mathrm{hr}$ in the presence of ATP alone (lane 1), a partially purified ISWI fraction alone (lane 3) or both ATP and the ISWI fraction (lane 2) followed by MNase digestion. The MNase digestion pattern was vizualized by ethidium bromide staining of agarose gels. The ability of the ISWI fraction and an approximately equal molar amount BRM complex (about 40 fmoles) to order a nucleosomal array was compared in a similar experiment containing an approximated 180-fold molar excess of nucleosomes (lanes 4-6). Protein amounts were estimated by comparison of silver and Coomassie-stained SDS-PAGE gels containing known amounts of marker proteins and quantitative Western blotting (data not shown). The input template was incubated in the presence of ATP and either no remodeling factor (lane 4), the ISWI fraction (lanes 5), or the BRM complex (monoS \#42) (lanes 6) and analyzed by MNase digestion.

results show that the BRM complex and ISWI factors display distinct functional properties.

\section{Zeste directly contacts specific BAPs}

Our results so far have established that the BRM complex is a transcriptional coactivator of Zeste. We now turn to the question of how the BRM complex is targeted. One attractive possibility is that Zeste directly binds to the BRM complex and recruits it to the DNA. To obtain more evidence for such a scenario, we performed a number of protein-protein interaction experiments. First, we determined whether Zeste can directly contact the BRM complex. An affinity resin was generated by immobilization of the purified BRM complex on beads coated with anti-BRM antibodies. As shown in Figure $6 \mathrm{~A}$, in vitro translated radiolabeled Zeste efficiently bound to the BRM complex affinity resin, but not to the control beads or to immunopurified ISWI complexes.

We next investigated which components of the BRM complex might be recognized by Zeste. To address this question we performed a far-Western experiment in which the BAPs were separated by SDS-PAGE, and transferred to a nitrocellulose membrane. Following denaturation and renaturation, the membrane was probed with 
A
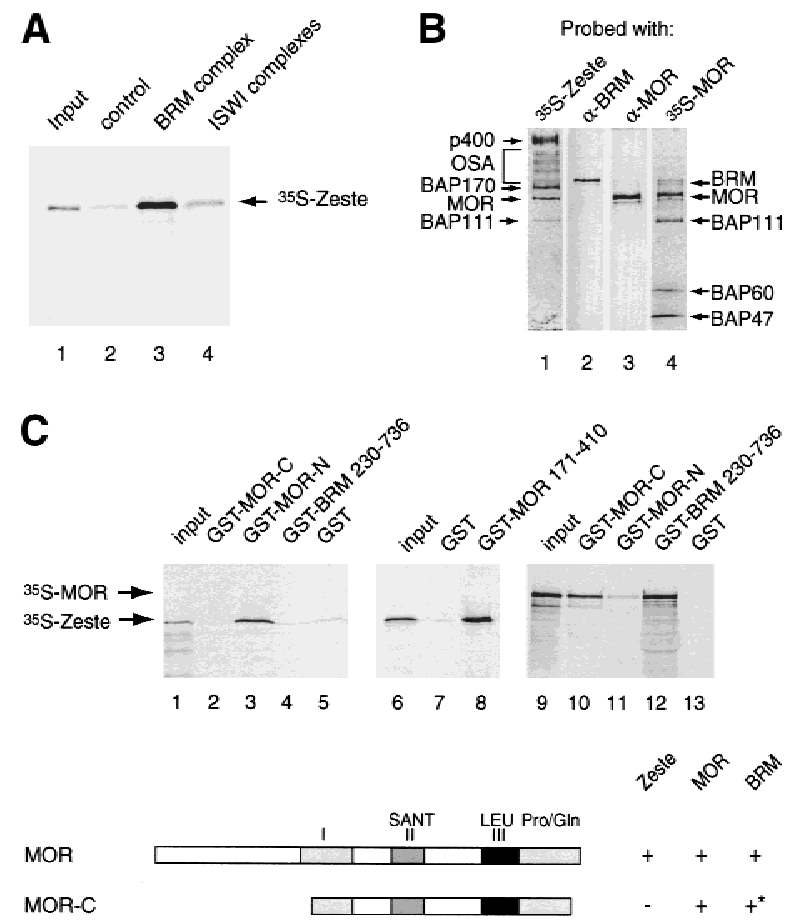

MOR-N

MOR $171-410$

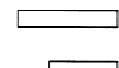

Figure 6. Zeste directly binds selected BAPs within the BRM complex. $(A)$ Zeste interacts with the BRM complex. Protein-A Sepharose resin (control, lane 2), anti-BRM affinity beads loaded with BRM complex (lane 3), or anti-ISWI affinity beads loaded ISWI complexes (lane 4) were incubated with ${ }^{35}$ S-labeled reticulocyte expressed Zeste. Protein complexes were washed, resolved by SDS-PAGE, and bound Zeste was detected by autoradiography. Lane 1 represents $5 \%$ of the input material used in the binding reactions. (B) Far-Western blotting analysis reveals that Zeste targets specific BAPs. The purified BRM complex (monoS \#42) was resolved by SDS-PAGE and transferred to nitrocellulose. The nitrocellulose membrane was treated with $6 \mathrm{M}$ guanidine- $\mathrm{HCl}$, renatured, washed, and incubated with ${ }^{35} \mathrm{~S}$-labeled reticulocyte expressed Zeste (lane 1) or MOR (lane 4). After extensive washing the filter was exposed to film. Filter 1 and 4 were reprobed with antibodies directed against BRM (lane 2) or MOR (lane 3), respectively. The positions of the BAPs bound by Zeste or MOR are indicated on the left (Zeste) or right (MOR) of the panels. (C) Mapping of the Zeste binding domain of MOR by GST pull-down assays. GST-MOR carboxyl terminus (residues 454-1174; lanes 2,10), GST-MOR amino terminus (residues 106-410; lanes 3,11), GST-MOR (residues 171-410; lane 8), GST-BRM (residues 230-736; lanes 4,12), or GST alone $($ lanes $5,7,13)$ were immobilized on glutathione-Sepharose beads and incubated with either ${ }^{35}$ S-Zeste (lanes $2-8$ ) or ${ }^{35}$ S-MOR (lanes 10-13). Protein complexes were washed, resolved by SDSPAGE and bound proteins were detected by autoradiography. Lanes 1, 6, and 9 represent $5 \%$ of the input material used in the binding reactions. The domain structure of MOR and its interaction domains with itself, BRM (asterisk indicates data from Crosby et al. 1999) and Zeste are indicated.

radiolabeled Zeste (Fig. 6B). Autoradiography of the blot revealed that Zeste directly bound to a number of BAPs, most notably MOR, BAP170, and p400. Somewhat less prominent binding was observed to BAP111 and OSA. When radiolabeled MOR was used as a probe in a farWestern assay, the pattern of binding was very different (Fig. 6B, lane 4). MOR bound to BRM and itself, as was previously reported (Crosby et al. 1999), as well as to BAP111, BAP60 and to either BAP47 or BAP45. The identity of BRM (Fig. 6B, lane 2), MOR (Fig. 6B, lane 3), and OSA (not shown) was confirmed by reprobing the blots with antibodies directed against these proteins. We conclude that Zeste and MOR contact distinct, partially overlapping sets of BAPs within the BRM complex.

Because MOR appeared to be a prominent target for Zeste and in vivo results have shown that they regulate some common genes such as Ubx (Laney and Biggin 1992; Brizuela and Kennison 1997), we decided to study the interaction between these two proteins in more detail. We expressed and purified GST-fusion proteins containing the amino-terminal third of MOR, the carboxyterminal two thirds of MOR, or a major portion of BRM (Crosby et al. 1999). The fusion proteins were immobilized on glutathione-Sepharose beads and incubated with full-length radiolabeled Zeste. Next, bound protein was analyzed by SDS-PAGE followed by autoradiography. Zeste efficiently bound to the amino-terminal third of MOR, but not to the remainder of the protein nor to BRM or GST alone (Fig. 6C, lanes 1-5). We were able to further narrow the Zeste binding domain of MOR to amino acids 171 to 410 (Fig. 6C, lanes 6-8). The binding pattern of radiolabeled MOR to these affinity resins was strikingly different from Zeste. MOR interacted with BRM and its own carboxy-terminal portion but not with the region of MOR targeted by Zeste (Fig. 6C, lanes 9-13). Thus, the domain of MOR that mediates the interaction with the activator Zeste is distinct from the protein regions responsible for its incorporation into the BRM complex. Taken together, these results show that Zeste can directly target specific subunits of the BRM complex, including MOR.

\section{Zeste binds MOR via a leucine zipper}

Having established that MOR is a target for Zeste, we next set out to identify the domain of Zeste that mediates this interaction. Zeste contains an amino-terminal helix-turn-helix type DNA binding domain and a carboxy-terminal extended leucine zipper that mediates multimerization and is essential for Zeste binding to its response elements (Chen and Pirotta 1993). Additionally, Zeste has regions that are characterized by a preponderance of particular amino acids such as acidic residues, glutamines, and alanines $(\mathrm{Q} / \mathrm{A})$ and prolines $(\mathrm{P}$ rich). A series of Zeste deletion mutants was generated and expressed using an in vitro transcription and translation system. Binding of distinct radiolabeled Zeste polypeptides to MOR was tested by GST pull-down experiments similar to those described above. We found that the leucine-zipper domain (residues 501-575) of Zeste was necessary and sufficient for binding to MOR (Fig. 7). As expected, neither the leucine zipper domain nor any of the other Zeste deletion mutants bound to 
Kal et al.

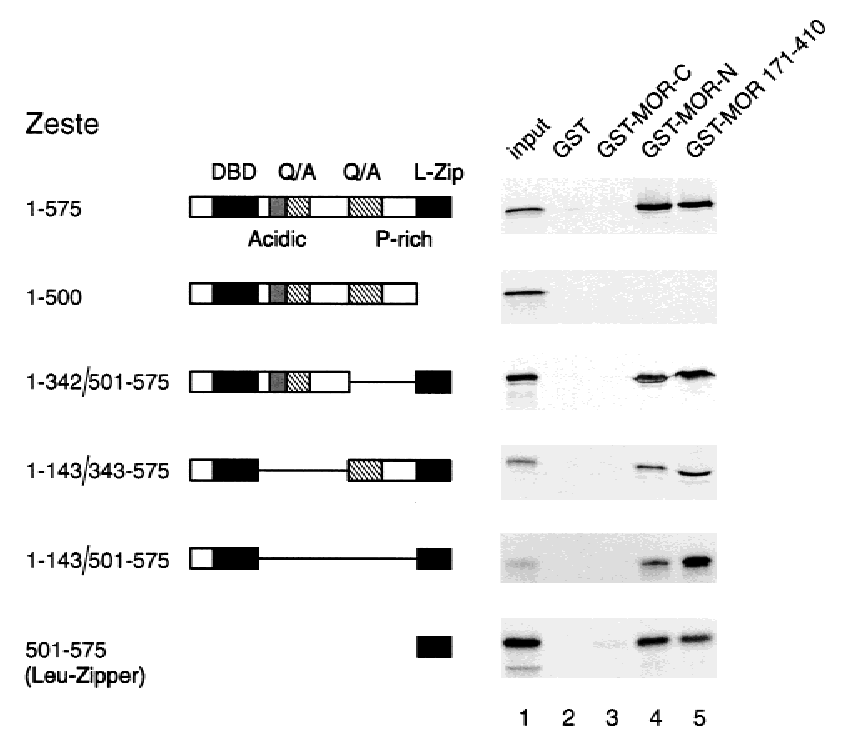

Figure 7. The leucine zipper of Zeste binds MOR. Mapping of the MOR-binding domain of Zeste by GST pull-down assays. GST alone (lane 2), GST-MOR carboxyl terminus (lane 3), GSTMOR amino terminus (lane 4), or GST-MOR residues 171-410 (lane 5) were immobilized on glutathione-Sepharose beads and incubated with ${ }^{35} \mathrm{~S}$-methionine-labeled Zeste or various Zeste deletion mutants. Protein complexes were washed, resolved by SDS-PAGE, and bound proteins were detected by autoradiography. Lane 1 represents $5 \%$ of the input material used in the binding reactions. The domain structure of Zeste and the amino acid residues present in the various deletion mutants are indicated (DBD) DNA-binding domain; (L-zip) leucine zipper; (Q/A) region rich in glutamines and alanines; $(\mathrm{AD})$ acidic domain; (P-rich) proline rich domain.

GST alone or the carboxy-terminal two thirds of MOR involved in MOR multimerization. Although a fusion of the DNA-binding domain with the leucine zipper bound DNA efficiently, it failed to activate transcription, indicating that additional domains of Zeste are needed for transcriptional activation (unpubl.). We conclude that Zeste targets the MOR subunit of the BRM complex via its leucine zipper domain that is also required for Zeste multimerization and DNA-binding.

\section{Discussion}

It has become evident that the modulation of chromatin structure is a major component of the mechanism of gene regulation. Two important unresolved questions are the targeting of remodeling factors and their functional specialization. Here, we have studied the cofactor requirements of a specialized transcriptional regulator, the trxG protein Zeste. Our results show that the BRM complex is an essential coactivator for Zeste-directed transcription on chromatin templates. Zeste can recruit the BRM complex via direct binding to specific BAPs. In contrast to BRM, the ISWI-containing remodelers are not required for Zeste activation. However, the ISWI factors are more potent spacing factors than the BRM complex.
These results support the notion that different remodeling complexes perform distinct functions.

\section{Zeste recruits the BRM coactivator complex}

Our data reveal that transcriptional activation on chromatin by Zeste is dependent on the BRM complex. Zeste directly targets the BRM complex via specific contacts with selected BAPs (Fig. 8). We found that the leucine zipper of Zeste, that is also essential for multimerization and DNA binding, mediates binding to the MOR subunit of the BRM complex. The relevance of the Zeste-MOR interaction is underscored by the in vivo requirement for MOR in normal expression of at least some genes activated by Zeste such as Ubx (Laney and Biggin 1992; Brizuela and Kennison 1997). The BRM complex is not required for promoter binding by Zeste, suggesting that it functions at a later step during the transcription cycle. Restructuring of the local chromatin environment by the recruited BRM complex may allow for the subsequent recruitment of other coactivators and the transcription machinery. In yeast cells, such an ordered recruitment has been observed at the $\mathrm{HO}$ promoter (Cosma et al. 1999; Yudkovsky et al. 1999). Finally, the notion that Zeste recruits the BRM complex is further supported by the catalytic amounts of BRM complex needed to mediate Zeste-directed transcription. We estimated a BRMto-nucleosome molar ratio of less than 1:50. Recently,

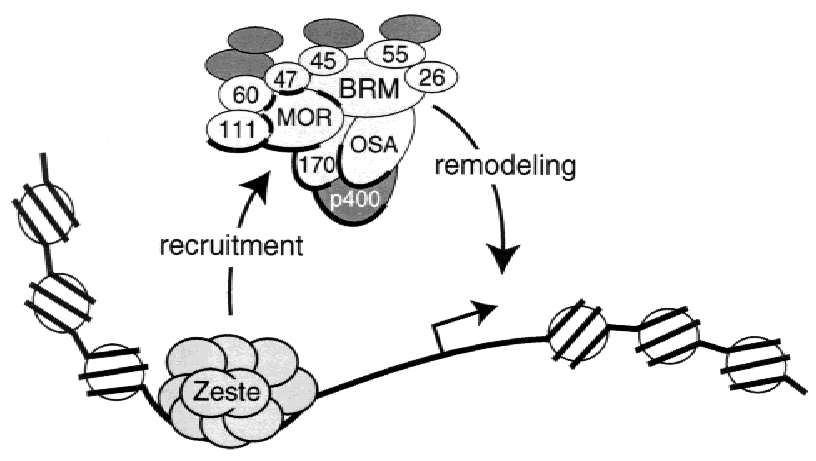

Figure 8. Summary and model. Our results suggest that the core BRM complex comprise 10 subunits (light grey) including trxG protein OSA and two putative novel BAPs of 170 and 26 $\mathrm{kD}$. More loosely associated factors (dark grey) that are not part of the core complex such as p400, are indicated. Zeste binds to multiple sites in the promoter DNA as a large oligomer and directly binds selective subunits within the BRM complex (indicated by a bold outline). These include most significantly MOR, BAP170, and p400, whereas somewhat less strong binding to OSA and BAP111 was observed. MOR interacts with itself and is assembled into the complex via binding to BRM, BAP111, BAP60, and either (the most likely candidate) BAP47 or BAP45. Contacts between MOR and other BAPs are indicated by a bold interface. All other contacts depicted here are speculative. The results from our experiments suggest that the BRM complex is an essential coactivator that can be recruited to specific genes by Zeste. The BRM complex may create an open chromatin conformation, here indicated as naked DNA, that could facilitate the docking of other transcription factors. 
direct recruitment of the yeast SWI/SNF complex by an acidic activation domain was reported (Neely et al. 1999|. Because Zeste contacts the BRM complex through different protein motives, it will be of interest to determine what subunits of the yeast SWI/SNF complex are contacted by acidic activators. This may establish whether different activators target distinct subunits in SWI/SNF-type remodeling complexes.

Can we generalize the recruitment mechanism we describe here? Although the majority of PcG and trxG proteins associate with specific chromosomal sites, they do not appear to bind DNA directly. Response elements for PcG and trxG proteins (PREs) are poorly defined sequences of several hundred base pairs (Pirrotta 1998; Lyko and Paro 1999). In addition to Zeste, there are a few candidate sequence-specific tethering factors such as pleiohomeotic and GAGA (Strutt et al. 1997; Brown et al. 1998; Fritsch et al. 1999). Thus far it has been impossible to reduce PREs to a number of simple sequence motives, therefore it is likely that there will be additional DNA-binding proteins that function as anchors for PcG and trxG proteins.

\section{A biochemical link between distinct trx $G$ proteins}

Zeste and BRM both belong to the trxG proteins that have been identified as transregulators of homeotic gene function in Drosophila (Kennison 1995). The majority of BAPs are not encoded by trxG genes and several other trxG proteins have been found to be part of separate protein complexes (Papoulas et al. 1998). Thus, it was unclear whether distinct trxG proteins may cooperate in a single biochemical pathway. Our study now establishes a direct physical interaction between four distinct trxG proteins during transcriptional activation. Previous analysis of osa and mor revealed a strong genetic interaction of these genes with brm (Brizuela and Kennison 1997; Vazquez et al. 1999). We show here that MOR and OSA are integral constituents of the BRM complex that are directly contacted by Zeste. While this paper was under review, Collins et al. (1999) also reported that OSA associates with the BRM complex. Genetic studies have indicated that MOR, OSA, BRM, and Zeste share at least some target genes (Biggin et al. 1988; Laney and Biggin 1992; Tamkun et al. 1992; Brizuela and Kennison 1997; Vazquez et al. 1999). Our results now provide a biochemical basis for the functional relationship between these trxG proteins.

Purification of the BRM complex and stringent coimmunoprecipitation experiments suggested the presence of two novel core BAPs in addition to OSA: BAP170 and BAP26. Moreover, it appeared that there are several less tightly associated proteins. We favor the idea that the interaction of the majority of these proteins with the core BRM complex is specific, because they copurify over several columns and remain associated during selective coimmunoprecipitation. Moreover, Zeste specifically interacts with the p400 protein, supporting the notion that its association with the BRM complex is functional.
Functional specialization of chromatin remodeling factors

An intersting question is whether distinct remodeling factors perform different functions or whether they are redundant? A number of recent studies shed light on the basic mechanisms by which SWI/SNF and ISWI remodelers catalyze nucleosome mobilization (Côté et al. 1998; Lorch et al. 1998, 1999; Schnitzler et al. 1998; BazettJones et al. 1999; Hamiche et al. 1999; Kingston and Narlikar 1999; Längst et al. 1999; Travers 1999; Whitehouse et al. 1999). However, their potential roles as regulators of transcription are still poorly understood. Here, we present a clear example of functional differentiation among chromatin remodelers. The BRM complex is an essential coactivator for Zeste, whereas the ISWI family members are not required. Reversibly, at least some ISWI remodeling factors appear to be more efficient at ordering nonperiodic nucleosomal arrays than the BRM complex. Thus, our side-by-side comparison of distinct endogenous Drosophila remodeling fac-tors shows that each performs distinct specialized functions.

As shown here and in other studies, the SWI/SNF-type remodelers appear to function in a highly selective manner. For example, the human SWI/SNF-related chromatin-remodeling complex, E-RC1 is required for the activation of the $\beta$-globin gene by the activator EKLF but does not work with another transcription factor, TFE3. (Armstrong et al. 1998). Moreover, it is pertinent to note that the yeast and Drosophila SWI/SNF family members were first identified by genetic screens for gene-specific regulators (Winston and Carlson 1992; Tamkun 1995). Thus, studies in yeast, mammals, and Drosophila all point to an integral and essential role for SWI/SNF remodelers in gene-specific transcriptional regulation. Although the ISWI remodelers are not required for Zeste function, they have been implicated in transcriptional activation by other regulators such as GAL4-VP16 (Ito et al. 1997; Mizuguchi et al. 1997; LeRoy et al. 1998; Di Croce et al. 1999). Several lines of evidence suggest that ISWI remodelers may act by a mechanism that is fundamentally distinct from that of the SWI/SNF family complexes. For example, unlike NURF, SWI/SNF does not seem to require the histone tails for remodeling (Georgel et al. 1997; Guyon et al. 1999|. Moreover, studies on NURF suggest that it remodels chromatin in a transient nonspecific manner, creating an opportunity for transcriptional activators to bind DNA (Mizuguchi et al. 1997). Such a mode of action does not involve the direct physical interactions between remodeler and activator we described here for the BRM complex. In conclusion, all available evidence points to an extensive functional specialization of ATPdependent chromatin remodeling factors. An attractive possibility is that different genes require the action of distinct subsets of remodeling complexes, histone acetyl transferases, and other coactivators. Such a combinatorial arrangement would vastly expand a cell's potential for precise and coordinated regulation of individual genes. 


\section{Materials and methods}

DNA constructs

Details of cloning procedures are available upon request. Briefly, using a PCR-based strategy a sequence encoding the FLAG-epitope was added at the end of the Zeste coding sequence and cloned into a modified version of the shuttle vector pVL1392 (Pharmingen) to generate pAK98. pAK156, used for transcription experiments, contains five Zeste binding sites, loosely based on the Ubx promoter, in front of a TATA-box containing a core promoter in a pBluescript backbone (Stratagene). For expression of GST-fusion proteins, indicated regions of MOR and BRM were cloned in pGEX1 (Pharmacia) (see also Crosby et al. 1999|. For in vitro transcripition/translation of MOR and Zeste, their coding sequences were cloned in pBluescript and pcDNA3.1/HisC (Invitrogen), respectively.

\section{Protein purifications}

Recombinant Zeste containing a carboxy-terminal FLAG-epitope was expressed in Sf9 cells using the baculovirus expression system and immunopurified using anti-FLAG M2 beads (Sigma) essentially as described by Chalkley and Verrijzer (1999).

Details of the protein purification procedures will be provided upon request. Briefly, all protein procedures were carried out at $4^{\circ} \mathrm{C}$ or on ice using HEMG buffer [ $25 \mathrm{mM}$ HEPES-KOH $(\mathrm{pH} 7.6)$, $0.1 \mathrm{~mm}$ EDTA, $12.5 \mathrm{~mm} \mathrm{MgCl}_{2}, 10 \%$ glycerol, $1 \mathrm{~mm}$ DTT, 0.2 mM AEBSF, and $1 \mu \mathrm{M}$ pepstatin, $0.01 \%$ Nonidet P-40 (NP-40)] containg varying amounts of $\mathrm{KCl}$. Nuclear extract derived from $500 \mathrm{~g}$ of Drosophila embryos (0-12 hr) were prepared and concentrated by POROS-Heparin (PerSeptive Biosystems) chromatography essentially as described by Heberlein and Tjian (1988). The heparin $400 \mathrm{~mm} \mathrm{KCl}$ fractions (HO.4) contained the vast majority of BRM, MOR, ISWI, and general transcription factors present in the nuclear extract. BRM complex and ISWI was further purified guided by Western blot analysis with antibodies directed against these proteins using a scheme based on that developed for the purification of RNA polymerase II general factors (Austin and Biggin 1996). The H0.4 fraction was loaded onto an $800 \mathrm{ml}$ Sephacryl S-300 column $\left(V_{\mathrm{e}}=300 \mathrm{ml}\right)$ (Pharmacia), equilibrated and developed with HEMG $100 \mathrm{mM} \mathrm{KCl}$. BRM eluted in the void, whereas the majority of ISWI eluted in later fractions. BRM and ISWI were further purified seperately on Bioscale Q10 (BioRad) and monoS (5/5) (Pharmacia) columns, developed with a linear salt gradient. The majority of BRM eluted from the Q10 at $225 \mathrm{~mm} \mathrm{KCl}$ and from the monoS at 270 $\mathrm{mm} \mathrm{KCl}$. ISWI eluted from the Q10 at $\sim 290 \mathrm{~mm} \mathrm{KCl}$ and from the monoS at $\sim 300 \mathrm{~mm} \mathrm{KCl}$. Western blot analysis using antiBRM and anti-ISWI antibodies as well as a comparison of silver staining patterns of SDS-PAGE gels established that the BRM fraction was free of ISWI and, likewise, the ISWI preparation did not contain BRM. Relative protein amounts were estimated by silver staining of SDS-PAGE gels and quantitative Western blot analysis.

\section{Chromatin procedures}

Drosophila core histones were purified from fly embryo nuclear extracts essentially as described by Bulger and Kadonaga (1994). The S-190 assembly extract was prepared and used for chromatin assembly as described by Bulger and Kadonaga (1994) and Kamakaka et al. (1993). For purposes of in vitro transcription the assembled chromatin was treated with sarkosyl $(0.05 \%$ final concentration for $5 \mathrm{~min}$ ) prior to purification by sucrose gradient sedimentation.
For chromatin assembly by salt dialysis, $50 \mu \mathrm{g}$ of plasmid was mixed with $50 \mu \mathrm{g}$ of purified Drosophila core histone proteins in $500 \mu$ l R-buffer [10 mM HEPES (pH 7.6), $1.5 \mathrm{~mm} \mathrm{MgCl}_{2}, 0.5 \mathrm{~mm}$ EGTA, $10 \%$ glycerol, $10 \mathrm{~mm} \beta$-glycerophosphate, $1 \mathrm{~mm} \mathrm{DTT}$, $0.2 \mathrm{~mm}$ PMSF] containing $2 \mathrm{M} \mathrm{KCl}$. The salt concentration was then reduced by three dialysis steps for $45 \mathrm{~min}$ against R-buffer containing $800 \mathrm{mM}, 150 \mathrm{~mm}$, and $10 \mathrm{~mm} \mathrm{KCl}$, respectively, in a microdialysis apparatus. Sucrose gradient sedimentation was used to separate naked DNA from chromatin. The input material for the spacing assay (Fig. 5) was prepared via a similar salt dialysis procedure in which we reduced the dialysis time to obtain arrays with a suboptimal spacing. Spacing activities of purified ISWI and BRM complexes were analysed after $1 \mathrm{hr}$ incubation at $27^{\circ} \mathrm{C}$ under standard assembly conditions (Bulger and Kadonaga 1994).

For purification, chromatin was loaded onto $20 \%$ to $50 \%$ sucrose gradients followed by ultracentrifugation in a SW41 rotor for $16 \mathrm{hr}$ at $26,000 \mathrm{rpm}$ at $4^{\circ} \mathrm{C}$. The chromatin containing fractions were collected and dialyzed against $\mathrm{R}$ buffer. The concentration of DNA in the collected fractions was estimated by ethidium bromide staining of agarose gels and compared with DNA standards. No BRM or ISWI could be detected by Western blot analysis of the chromatin template (data not shown).

The analysis of chromatin templates by partial digestion with MNase and Southern blotting was performed essentially as described (Bulger and Kadonaga 1994). For remodeling assays, purified Zeste protein was added to the assembled chromatin and incubated for $30 \mathrm{~min}$ prior to MNase digestion. Blots were first probed with a labeled oligonucleotide corresponding to the promoter, and following radiography, stripped and reprobed with an oligonucleotide corresponding to the ampicillin resistance gene (distal probe). DNaseI footprinting was performed essentially as described previously (Gralla 1985).

\section{In vitro transcription}

Transcription reactions and primer extension analysis were carried out essentially as described by Kadonaga (1990). The template was plasmid pAK156 that contained five Zeste binding sites upstream of a consensus TATA box and Inr element. Transcription reactions were performed in a total volume of $25 \mu \mathrm{l}$ and contained $100 \mathrm{ng}$ of template DNA (in the form of either S-190 chromatin, salt dialysis chromatin or naked DNA), $40 \mathrm{~mm}$ HEPES-KOH (pH 7.6), $40 \mathrm{~mm} \mathrm{KCl,} 1 \%$ polyvinyl alcohol (molecular mass 10.000), $0.1 \mu \mathrm{l}$ RNase inhibitor, $0.8 \mathrm{~mm}$ DTT. The transcription machinery and remodeling factors were provided by addition of $2 \mu \mathrm{l} \mathrm{H0.4}$ or immunodepleted H0.4. Upon addition of Zeste and/or 0.6 mM ATP, reactions were incubated for $20 \mathrm{~min}$ at room temperature prior to the start of transcription by the addition of $0.6 \mathrm{~mm}$ each of CTP, GTP, and UTP. After 30 min at $30^{\circ} \mathrm{C}$ transcription was stopped by the addition of $100 \mu \mathrm{l}$ stop buffer (20 mM EDTA, 1\% SDS, $0.2 \mathrm{M} \mathrm{NaCl}, 0.25 \mathrm{mg} / \mathrm{ml}$ yeast RNA, $50 \mu \mathrm{g} / \mathrm{ml}$ Proteinase K). The RNA transcripts were visualized by primer extension using a radiolabeled primer (PV313) and analyzed on an 8\% denaturing polyacrylamide gel.

\section{Antibodies, immunoprecipitations, and immunodepletions}

All immunologic procedures were performed essentially as described previously (Harlow and Lane 1988). The anti-BRM and anti-ISWI antisera were generated by immunization of rabbits with peptides corresponding to either BRM (MASPSPANSPMPPPQ) or to ISWI (MSKTDTAAVEATEEN) protein sequences, coupled to keyhole limpet haemocyanin (KLH). Western blots and immunoprecipitations were confirmed with antibodies directed against other parts of BRM or ISWI, respec- 
tively (data not shown). Antibodies were affinity purified as described by Hancock and Evan (1998). The rabbit anti-dNAP1 antisera was generated against bacterially expressed dNAP1 (A. Kal, unpubl.), anti-MOR antibodies (Crosby et al. 1999), and anti-OSA monoclonal antibodies (Treisman et al. 1997) have been described.

For immunoprecipitations and immunodepletions, affinity purified antibodies were cross-linked to protein-A beads (Pharmacia) using dimethylpimelimidate as described (Harlow and Lane 1988). For immunoprecipitations, affinity resins were incubated with protein fractions for $2 \mathrm{hr}$ at $4^{\circ} \mathrm{C}$ in HEMG buffer containing $225 \mathrm{~mm} \mathrm{KCl}$. Following extensively washes with excess HEMG containg $\mathrm{KCl}$ as indicated in the text, bound proteins were eluted in an SDS-sample buffer, resolved by SDSPAGE, and vizualized by silver staining or Western blotting. For immunodepletions, the H0.4 or S-190 fraction was incubated with affinity beads, equilibrated in HEMG $400 \mathrm{~mm} \mathrm{KCl}$ or Rbuffer, respectivily, for $2 \mathrm{hr}$ at $4^{\circ} \mathrm{C}$. The beads were removed by centrifugation and the fraction was reapplied to fresh beads for a second round of depletion.

\section{Protein-protein interactions}

For Zeste-BRM complex interactions, Protein-A Sepharose, anti-BRM or anti-ISWI affinity beads were incubated with the $\mathrm{H} 0.4$ fraction purified for $2 \mathrm{hr}$ at $4^{\circ} \mathrm{C}$ followed by extensive washes with HEMG containing $0.1 \mathrm{M} \mathrm{KCl}$. The affinity resins were incubated for $2 \mathrm{hr}$ with ${ }^{35} \mathrm{~S}$-labeled reticulocyte expressed Zeste, washed extensively with RIPA buffer (Jimenez et al. 1999), and analyzed by SDS-PAGE followed by autoradiography. The GST pulldown experiments were performed essentially as described by Jimenez et al. (1999). Far-Western analysis was performed as described by Kaelin et al. (1992) using ${ }^{35}$ S-labeled reticulocyte expressed Zeste or MOR. Following autoradiography to detect bound proteins, blots were reprobed with antibodies directed against MOR, BRM, or OSA to facilitate alignment with silver-stained gels and identification of interacting BAPs.

\section{Acknowledgments}

We are grateful to Jessica Treisman for anti-OSA antibodies; Jim Kadonaga, Lee Kraus, and Karen Robinson for generously instructing C.P.V. in chromatin procedures; Robert Tjian for support during the initiation of this work; Mark Biggin and Vincenzo Pirrotta for plasmids; Gillian Chalkley, Chaya Miller, Aline Richard, and Simon Vincent for technical assistance and Nicola O'Reilly for peptide synthesis. We also thank Jesper Svejstrup, David Ish-Horowicz and Richard Treisman for helpful discussions and Eric Kalkhoven, Katerina Katsani, Natalie Little and Jesper Svejstrup for critical reading of the manuscript.

The publication costs of this article were defrayed in part by payment of page charges. This article must therefore be hereby marked "advertisement" in accordance with 18 USC section 1734 solely to indicate this fact.

\section{References}

Armstrong, J.A. and B.M. Emerson. 1998. Transcription of chromatin: These are complex times. Curr. Opin. Genet. Dev. 8: $165-172$.

Armstrong, J.A., J.J. Bieker, and B.M. Emerson. 1998. A SWI/ SNF-related chromatin remodeling complex, E-RC1, is required for tissue-specific transcriptional regulation by EKLF in vitro. Cell 95: 93-104.

Austin, R.J. and M.D. Biggin. 1996. Purification of the Dro- sophila RNA polymerase II general transcription factors. Proc. Nat1. Acad. Sci. 93: 5788-5792.

Bazett-Jones, D.P., J. Côté, C.C. Landel, C.L. Peterson, and J.L. Workman. 1999. The SWI/SNF complex creates loop domains in DNA and polynucleosome arrays and can disrupt DNA-histone contacts within these domains. Mol. Cell. Biol. 19: 1470-1478.

Biggin, M.D., S. Bickel, M. Benson, V. Pirrotta, and R. Tjian. 1988. Zeste encodes a sequence-specific transcription factor that activates the Ultrabithorax promoter in vitro. Cell 53: 713-722.

Breen, T.R., V. Chinwalla, and P.J. Harte. 1995. Trithorax is required to maintain engrailed expression in a subset of engrailed-expressing cells. Mech. Dev. 52: 89-98.

Brizuela, B.J. and J.A. Kennison. 1997. The Drosophila homeotic gene moira regulates expression of engrailed and HOM genes in imaginal tissues. Mech. Dev. 65: 209-220.

Brown, J.L., D. Mucci, M. Whiteley, M.L. Dirksen, and J.A. Kassis. 1998. The Drosophila Polycomb group gene pleiohomeotic encodes a DNA-binding protein with homology to the transcription factor YY1. Mol. Cell 1: 1057-1064.

Bulger, M. and J.T. Kadonaga. 1994. Biochemical reconstitution of chromatin with physiological nucleosome spacing. Meth. Mol. Genet. 5: 241-262.

Cairns, B.R. 1998. Chromatin remodeling machines: similar motors, ulterior motives. Trends. Biochem. Sci. 23: 20-25.

Chalkley, G.E. and C.P. Verrijzer. 1999. DNA binding site selection by RNA polymerase II TAFs: A TAF(II)250$\mathrm{TAF}(\mathrm{II}) 150$ complex recognizes the initiator. EMBO $I$. 18: $4835-4845$.

Chen, J.D. and V. Pirrotta. 1993. Multimerization of the Drosophila zeste protein is required for efficient DNA binding. EMBO J. 12: 2075-2083.

Collins, R.T., T. Furukawa, N. Tanese and J.E. Treisman. 1999. Osa associates with the Brahma chromatin remodeling complex and promotes the activation of some target genes. EMBO T. 18: 7029-7040.

Corona, D.F., G. Längst, C.R. Clapier, E.J. Bonte, S. Ferrari, J.W. Tamkun, and P.B. Becker. 1999. ISWI is an ATP-dependent nucleosome remodeling factor. Mol. Cell 3: 239-245.

Cosma, M.P., T. Tanaka, and K. Nasmyth. 1999. Ordered recruitment of transcription and chromatin remodeling factors to a cell cycle- and developmentally regulated promoter. Cell 97: 299-311.

Côté, J., C.L. Peterson, and J.L. Workman. 1998. Perturbation of nucleosome core structure by the SWI/SNF complex persists after its detachment, enhancing subsequent transcription factor binding. Proc. Natl. Acad. Sci. 95: 4947-4952.

Crosby, M.A., C. Miller, T. Alon, K.L. Watson, C.P. Verrijzer, R. Goldman-Levi, and N.B. Zak. 1999. The trithorax group gene moira encodes a brahma-associated putative chromatin-remodeling factor in Drosophila melanogaster. Mol. Cell. Biol. 19: 1159-1170.

Di Croce, L., R. Koop, P. Venditti, H.M. Westphal, K.P. Nightingale, D.F. Corona, P.B. Becker, and M. Beato. 1999. Twostep synergism between the progesterone receptor and the DNA-binding domain of nuclear factor 1 on MMTV minichromosomes. Mol. Cell 4: 45-54.

Elfring, L.K., C. Daniel, O. Papoulas, R. Deuring, M. Sarte, S. Moseley, S.J. Beek, W.R. Waldrip, G. Daubresse, A. DePace et al. 1998. Genetic analysis of brahma: the Drosophila homolog of the yeast chromatin remodeling factor SWI2/SNF2. Genetics 148: 251-265.

Fritsch, C., J.L. Brown, J.A. Kassis, and J. Muller. 1999. The DNA-binding polycomb group protein pleiohomeotic mediates silencing of a drosophila homeotic gene. Development 
126: $3905-3913$.

Georgel, P.T., T. Tsukiyama, and C. Wu. 1997. Role of histone tails in nucleosome remodeling by Drosophila NURF. EMBO J. 16: 4717-4726.

Gralla, J.D. 1985. Rapid "footprinting" on supercoiled DNA. Proc. Nat1. Acad. Sci. 82: 3078-3081.

Guyon, J.R., G.J. Narlikar, S. Sif, and R.E. Kingston. 1999. Stable remodeling of tailless nucleosomes by the human SWI-SNF complex. Mol. Cell. Biol. 19: 2088-2097.

Hamiche, A., R. Sandaltzopoulos, D.A. Gdula, and C. Wu. 1999. ATP-dependent histone octamer sliding mediated by the chromatin remodeling complex NURF. Cell 97: 833-842.

Hancock, D.C. and G.I. Evan. 1998. Production and characterization of antibodies against synthetic peptides. Meth. Mol. Biol. 80: 15-22.

Harlow, E. and D. Lane. 1988. In Antibodies: A laboratory manual. Cold Spring Harbor Laboratory Press, Cold Spring Harbor, NY.

Heberlein, U. and R. Tjian. 1988. Temporal pattern of alcohol dehydrogenase gene transcription reproduced by Drosophila stage-specific embryonic extracts. Nature 331: 410-415.

Ito, T., M. Bulger, M.J. Pazin, R. Kobayashi, and J.T. Kadonaga. 1997. ACF, an ISWI-containing and ATP-utilizing chromatin assembly and remodeling factor. Cell 90: 145-155.

Ito, T., M.E. Levenstein, D.V. Fyodorov, A.K. Kutach, R. Kobayashi, and J.T. Kadonaga. 1999. ACF consists of two subunits, Acf1 and ISWI, that function cooperatively in the ATP-dependent catalysis of chromatin assembly. Genes \& Dev. 13: 1529-1539.

Jacobs, J.J., K. Kieboom, S. Marino, R.A. DePinho, and M. van Lohuizen. 1999. The oncogene and Polycomb-group gene bmi-1 regulates cell proliferation and senescence through the ink4a locus. Nature 397: 164-168.

Jimenez, G., C.P. Verrijzer, and D. Ish-Horowicz. 1999. A conserved motif in goosecoid mediates groucho-dependent repression in Drosophila embryos. Mol. Cell. Biol. 19: 20802087.

Judd, B.H. 1995. Mutations of zeste that mediate transvection are recessive enhancers of position-effect variegation in Drosophila melanogaster Genetics 141: 245-253.

Kadonaga, J.T. 1990. Assembly and disassembly of the Drosophila RNA polymerase II complex during transcription. J. Biol. Chem. 265: 2624-2631.

1998. Eukaryotic transcription: an interlaced network of transcription factors and chromatin-modifying machines. Cell 92: 307-313.

Kaelin, W.G.J., W. Krek, W.R. Sellers, J.A. DeCaprio, F. Ajchenbaum, C.S. Fuchs, T. Chittenden, Y. Li, P.J. Farnham, and M.A. Blanar. 1992. Expression cloning of a cDNA encoding a retinoblastoma-binding protein with E2F-like properties. Cell 70: 351-364.

Kamakaka, R.T., M. Bulger, and J.T. Kadonaga. 1993. Potentiation of RNA polymerase II transcription by Gal4-VP16 during but not after DNA replication and chromatin assembly. Genes \& Dev. 7: 1779-1795.

Katsani, K.R., M.A. Hajibagheri, and C.P. Verrijzer. 1999. Cooperative DNA binding by GAGA transcription factor requires the conserved $\mathrm{BTB} / \mathrm{POZ}$ domain and reorganizes promoter topology. EMBO J. 18: 698-708.

Kennison, J.A. 1995. The Polycomb and trithorax group proteins of Drosophila: trans-regulators of homeotic gene function. Annu. Rev. Genet. 29: 289-303.

Kennison, J.A. and J.W. Tamkun. 1988. Dosage-dependent modifiers of polycomb and antennapedia mutations in Drosophila. Proc. Natl. Acad. Sci. 85: 8136-8140.

Kingston, R.E. and G.J. Narlikar. 1999. ATP-dependent remod- eling and acetylation as regulators of chromatin fluidity. Genes \& Dev. 13: 2339-2352.

Kingston, R.E., C.A. Bunker, and A.N. Imbalzano. 1996. Repression and activation by multiprotein complexes that alter chromatin structure. Genes \& Dev. 10: 905-920.

Kornberg, R.D. and Y. Lorch. 1999. Twenty-five years of the nucleosome, fundamental particle of the eukaryote chromosome. Cell 98: 285-294.

Laney, J.D. and M.D. Biggin. 1992. Zeste, a nonessential gene, potently activates Ultrabithorax transcription in the Drosophila embryo. Genes \& Dev. 6: 1531-1541.

Längst, G., E.J. Bonte, D.F. Corona, and P.B. Becker. 1999. Nucleosome movement by CHRAC and ISWI without disruption or trans-displacement of the histone octamer. Cell 97: 843-852.

LeRoy, G., G. Orphanides, W.S. Lane, and D. Reinberg. 1998. Requirement of RSF and FACT for transcription of chromatin templates in vitro. Science 282: 1900-1904.

Lorch, Y., B.R. Cairns, M. Zhang, and R.D. Kornberg. 1998. Activated RSC-nucleosome complex and persistently altered form of the nucleosome. Cell 94: 29-34.

Lorch, Y., M. Zhang, and R.D. Kornberg. 1999. Histone octamer transfer by a chromatin-remodeling complex. Cell 96: 389392.

Lyko, F. and R. Paro. 1999. Chromosomal elements conferring epigenetic inheritance. Bioessays 21: 824-832.

McGinnis, W. and R. Krumlauf. 1992. Homeobox genes and axial patterning. Cell 68: 283-302.

Mizuguchi, G., T. Tsukiyama, J. Wisniewski, and C. Wu. 1997. Role of nucleosome remodeling factor NURF in transcriptional activation of chromatin. Mol. Cell 1: 141-150.

Neely, K.E., A.H. Hassan, A.E. Wallberg, D.J. Steger, B.R. Cairns, A.P. Wright, and J.L. Workman. 1999. Activation domain-mediated targeting of the SWI/SNF complex to promoters stimulates transcription from nucleosome arrays. Mol. Cell 4: 649-655.

Papoulas, O., S.J. Beek, S.L. Moseley, C.M. McCallum, M. Sarte, A. Shearn, and J.W. Tamkun. 1998. The Drosophila trithorax group proteins BRM, ASH1 and ASH2 are subunits of distinct protein complexes. Development 125: 3955-3966.

Paro, R. 1995. Propagating memory of transcriptional states. Trends Genet. 11: 295-297.

Pelegri, F. and R. Lehmann. 1994. A role of polycomb group genes in the regulation of gap gene expression in Drosophila. Genetics 136: 1341-1353.

Phelan, M.L., S. Sif, G.J. Narlikar, and R.E. Kingston. 1999. Reconstitution of a core chromatin remodeling complex from SWI/SNF subunits. Mol. Cell 3: 247-253.

Phillips, M.D. and A. Shearn. 1990. Mutations in polycombeotic, a Drosophila polycomb-group gene, cause a wide range of maternal and zygotic phenotypes. Genetics 125: 91-101.

Pirrotta, V. 1998. Polycombing the genome: PcG, trxG, and chromatin silencing. Cell 93: 333-336.

Pirrotta, V., S. Bickel, and C. Mariani. 1988. Developmental expression of the Drosophila zeste gene and localization of zeste protein on polytene chromosomes. Genes \& Dev. 2: $1839-1850$.

Rastelli, L., C.S. Chan, and V. Pirrotta. 1993. Related chromosome binding sites for zeste, suppressors of zeste and Polycomb group proteins in Drosophila and their dependence on enhancer of zeste function. EMBO J. 12: 1513-1522.

Rosen, C., D. Dorsett, and J. Jack. 1998. A proline-rich region in the Zeste protein essential for transvection and white repression by Zeste. Genetics 148: 1865-1874.

Schnitzler, G., S. Sif, and R.E. Kingston. 1998. Human SWI/SNF interconverts a nucleosome between its base state and a 
stable remodeled state. Cell 94: 17-27.

Shao, Z., F. Raible, R. Mollaaghababa, J.R. Guyon, C.T. Wu, W. Bender, and R.E. Kingston. 1999. Stabilization of chromatin structure by PRC1, a Polycomb complex. Cell 98: 37-46.

Strutt, H., G. Cavalli, and R. Paro. 1997. Co-localization of Polycomb protein and GAGA factor on regulatory elements responsible for the maintenance of homeotic gene expression. EMBO I. 16: 3621-3632.

Tamkun, J.W. 1995. The role of brahma and related proteins in transcription and development. Curr. Opin. Genet. Dev. 5: 473-477.

Tamkun, J. W., R. Deuring, M.P. Scott, M. Kissinger, A.M. Pattatucci, T.C. Kaufman, and J.A. Kennison. 1992. Brahma: a regulator of Drosophila homeotic genes structurally related to the yeast transcriptional activator SNF2/SWI2. Cell 68: $561-572$.

Travers, A. 1999. An engine for nucleosome remodeling. Cell 96: 311-314.

Treisman, J. E., A. Luk, G.M. Rubin, and U. Heberlein. 1997. Eyelid antagonizes wingless signaling during Drosophila development and has homology to the Bright family of DNAbinding proteins. Genes \& Dev. 11: 1949-1962.

Tsukiyama, T. and C. Wu. 1995. Purification and properties of an ATP-dependent nucleosome remodeling factor. Cell 83: $1011-1020$.

. 1997. Chromatin remodeling and transcription. Curr. Opin. Genet. Dev. 7: 182-191.

Tsukiyama, T., J. Palmer, C.C. Landel, J. Shiloach, and C. Wu. 1999. Characterization of the imitation switch subfamily of ATP-dependent chromatin-remodeling factors in Saccharomyces cerevisiae. Genes \& Dev. 13: 686-697.

Varga-Weisz, P.D. and P.B. Becker. 1998. Chromatin-remodeling factors: machines that regulate? Curr. Opin. Cell Biol. 10: 346-353.

Varga-Weisz, P.D., M. Wilm, E. Bonte, K. Dumas, M. Mann, and P.B. Becker. 1997. Chromatin-remodelling factor CHRAC contains the ATPases ISWI and topoisomerase II. Nature 388: 598-602.

Vazquez, M., L. Moore, and J.A. Kennison. 1999. The trithorax group gene osa encodes an ARID-domain protein that genetically interacts with the brahma chromatin-remodeling factor to regulate transcription. Development 126: 733-742.

Wade, P.A. and A.P. Wolffe. 1999. Transcriptional regulation: SWItching circuitry. Curr. Biol. 9: R221-R224.

Wang, W., Y. Xue, S. Zhou, A. Kuo, B.R. Cairns, and G.R. Crabtree. 1996. Diversity and specialization of mammalian SWI/ SNF complexes. Genes \& Dev. 10: 2117-2130.

Whitehouse, I., A. Flaus, B.R. Cairns, M.F. White, J.L. Workman, and T. Owen-Hughes. 1999. Nucleosome mobilization catalysed by the yeast SWI/SNF complex. Nature 400: 784 787.

Winston, F. and M. Carlson. 1992. Yeast SNF/SWI transcriptional activators and the SPT/SIN chromatin connection. Trends Genet. 8: 387-391.

Workman, J.L. and R.E. Kingston. 1998. Alteration of nucleosome structure as a mechanism of transcriptional regulation. Annu. Rev. Biochem. 67: 545-579.

Yudkovsky, N., C. Logie, S. Hahn, and C.L. Peterson. 1999. Recruitment of the SWI/SNF chromatin remodeling complex by transcriptional activators. Genes \& Dev. 13: 23692374. 


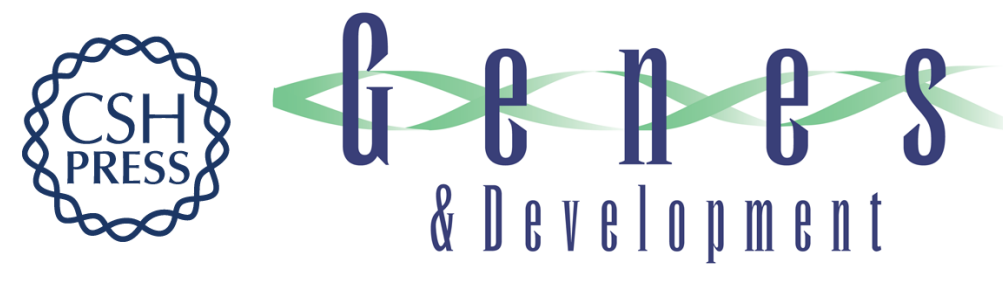

\section{The Drosophila Brahma complex is an essential coactivator for the trithorax group protein Zeste}

Arnoud J. Kal, Tokameh Mahmoudi, Naomi B. Zak, et al.

Genes Dev. 2000, 14:

Access the most recent version at doi:10.1101/gad.14.9.1058

References This article cites 77 articles, 33 of which can be accessed free at: http://genesdev.cshlp.org/content/14/9/1058.full.html\#ref-list-1

License

Email Alerting

Receive free email alerts when new articles cite this article - sign up in the box at the top Service right corner of the article or click here.

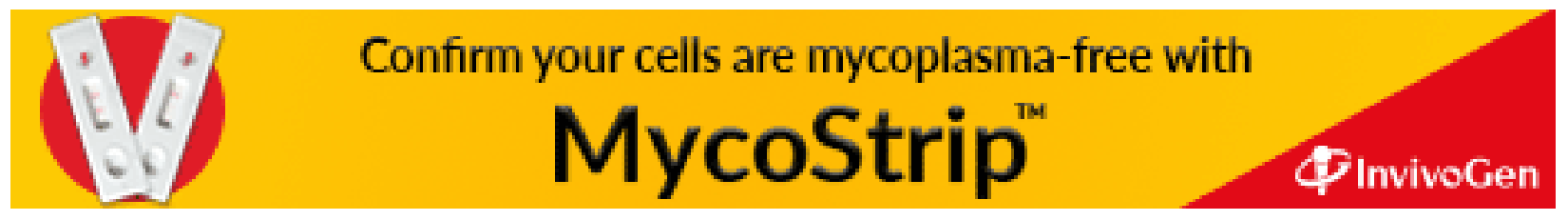

